Cite this: RSC Adv., 2015, 5, 23443

Received 18th January 2015

Accepted 25th February 2015

DOI: $10.1039 / \mathrm{c} 5 \mathrm{ra01025j}$

www.rsc.org/advances

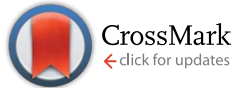

\section{Solvatochromic fluorescent BODIPY derivative as imaging agent in camptothecin loaded hexosomes for possible theranostic applications $\dagger$}

\author{
Claudia Caltagirone, ${ }^{\text {*a }}$ Massimiliano Arca, ${ }^{a}$ Angela M. Falchi, ${ }^{b}$ Vito Lippolis, ${ }^{a}$ \\ Valeria Meli, ${ }^{a}$ Maura Monduzzi, ${ }^{a}$ Tommy Nylander, ${ }^{c}$ Antonella Rosa, ${ }^{b}$ Judith Schmidt, ${ }^{d}$ \\ Yeshayahu Talmon ${ }^{d}$ and Sergio Murgia*a
}

\begin{abstract}
We here discuss the potential theranostic nanomedicine application of an innovative formulation consisting of monoolein-based nanoparticles with a two-dimensional hexagonal inner structure stabilized in water using a mixture of $\mathrm{PEO}_{132}-\mathrm{PPO}_{50}-\mathrm{PEO}_{132}$ block copolymers with and without conjugated folate for targeting. The proposed tumor-cell targeted formulation was shown to be able to simultaneously host the model anticancer drug camptothecin and a pyrene-modified BODIPY fluorophore, based on dynamic light scattering, small-angle $\mathrm{X}$-ray scattering, and cryogenic transmission electron microscopy. The photophysical properties of the fluorophore were studied in solution in various solvents. A marked fluorescent solvatochromism, whose origin was explained by time-dependent density functional theory theoretical calculations, was observed. Fluorescence microscopy showed that HeLa cells readily internalize these nanoparticles, and that the fluorophore localizes within the lipid droplets. In addition, cytotoxicity test revealed that these nanoparticles are not toxic at the concentration used for the imaging analysis.
\end{abstract}

\section{Introduction}

Polar lipids such as phospholipids or monoglycerides are amphiphilic in nature, therefore they self-assemble in aqueous solution to form a number of different liquid crystalline phases. Among others, monoolein (MO, glycerol monooleate) received much attention in the last three decades because of its rich structural polymorphism. ${ }^{1-3}$ Besides a reverse micellar phase found at very low water content, the MO binary phase diagram at $25{ }^{\circ} \mathrm{C}$ is characterized by a lamellar $\left(\mathrm{L}_{\alpha}\right)$ and two cubic bicontinuous liquid crystalline phases, namely, the gyroid $\left(\mathrm{C}_{\mathrm{G}}\right.$, space group Ia3d) and the double diamond $\left(\mathrm{C}_{\mathrm{D}}\right.$, space group $P n 3 m$ ). The last two are constituted by a curved, triply periodic, and non-intersecting MO bilayer folded to form two disjoint continuous water channels. Although the role of the $\mathrm{C}_{\mathrm{G}}$ and the $\mathrm{C}_{\mathrm{D}}$ phases in the cell machinery is not fully clarified, they were

${ }^{a}$ Dipartimento di Scienze Chimiche e Geologiche, Università degli Studi di Cagliari, S.S. 554 Bivio per Sestu, 09042, Monserrato, CA, Italy. E-mail: ccaltagirone@unicait; murgias@unica.it; Tel: +390706754452

${ }^{b}$ Dipartimento di Scienze Biomediche, Università di Cagliari, S.S. 554 Bivio Sestu, I-09042, Monserrato, CA, Italy

'Division of Physical Chemistry, Department of Chemistry, Center for Chemistry and Chemical Engineering, Lund University, P.O. Box 124, SE-221 00 Lund, Sweden

${ }^{d}$ Department of Chemical Engineering, Technion - Israel Institute of Technology, Haifa 32000, Israel

$\dagger$ Electronic supplementary information (ESI) available: Material and methods; synthesis of Py-BODIPY; details of DFT calculations. See DOI: 10.1039/c5ra01025j virtually found ubiquitous in nature, ${ }^{4}$ and several papers were devoted to shed some light on the biological function of this amazing nanostructures. ${ }^{5-7}$ Remarkably, the entrapment of lipophilic molecules into these cubic bicontinuous phases may alter the MO effective packing parameter, causing the evolution towards a reverse hexagonal phase ${ }^{8}$ (i.e., a bulk phase organized in water cylinders surrounded by a MO monolayer, and packed in a two-dimensional hexagonal array), while the local order and dynamics of the MO alkyl chain remains unaltered., ${ }^{9,10}$ The polymorphism of MO in water can be extended by adding small amounts of lauroylcholine chloride, which causes the formation of a very stable solution of small unilamellar vesicles. ${ }^{11,12}$ When increasing the dispersed phase content, they organize into a vesicle gel. ${ }^{13}$

As for lamellar phases that can be dispersed into vesicles, the cubic and hexagonal phases can be formulated into stable colloidal dispersion in water, known as cubosomes and hexosomes, respectively. In order to obtain mono-disperse formulations, a dispersion agent like the nonionic poly(ethylene oxide)poly(propylene oxide)-poly(ethylene oxide) (PEO-PPO-PEO) triblock copolymers, known as Pluronics, can be used..$^{14,15}$ These copolymers anchor their hydrophobic moiety in the nanoparticle, while protruding their hydrophilic segments into the solvent. This provides the particles with a PEO corona, which ensures a strong steric stabilization of the nanoparticles. Recently, such dispersions were proposed as platforms in theranostic nanomedicine. ${ }^{16-20}$ With the purpose of discovering and treating 
diseases at the earliest stage and with limited side effects, a new strategy to engineer different kinds of nanocarriers for the sustained, controlled, and targeted delivery of both therapeutic and diagnostic agents at the same time has emerged. ${ }^{21}$ Indeed, our group has demonstrated that, as with other kinds of nanoparticles, cubosomes can be simultaneously loaded with optical imaging agents and anticancer drugs. ${ }^{16,19}$ Cubosome and hexosome formulations possess a number of properties that can be exploited in nanomedicine but, most of all, (i) they have the right size for theranostic applications, and (ii) they are biodegradable. Moreover, these nanoparticles can be considered to be stealth particles, because their PEO corona is expected to prevent the adsorption of macromolecules on the nanoparticle surface. This prevents the formation of the biomolecular corona, thus reducing the clearance from the bloodstream via the mononuclear phagocytic system (MPS). ${ }^{22}$ When considering cubosomes and hexosomes for cancer diagnostic and therapy (as in the case discussed in this paper), it deserves also noticing that their size is within the range required for the tumor tissues passive targeting through the enhanced permeation mechanism (EPR).$^{23}$ We also proved that decoration of their surface with targeting moieties capable of addressing them at cancer cells is feasible, making these lipid-based nanoparticles even more appealing for theranostic nanomedicine applications in oncology. ${ }^{16}$

Over the past decade increasing efforts have been devoted to the synthesis of new organic chromophores or nanoparticles ${ }^{24,25}$ with strong absorption and emission bands for imaging applications, both in vitro and in vivo. In particular, considerable attention has been addressed towards the development of derivatives of 4,4-difluoro-4-bora-3a,4a-diaza-s-indacene (BODIPY) because of their high molar extinction coefficients and quantum yields, excellent photostability and high solubility in commonly used organic solvents. BODIPY derivatives have been included into different type of nanoparticles, ${ }^{26-33}$ belonging both to the hard and the soft matter realm.

Here, we report the formulation of MO-based hexosomes loaded with both the model chemotherapeutic drug camptothecin and a pyrene-modified BODIPY fluorophore (Py-BODIPY) also stabilized by a mixture of Pluronic F108 (PF108) and folateconjugated PF108 (PF108-FA), and investigate their physicochemical and photophysical properties. Theoretical calculations were used to elucidate the origin of the peculiar fluorescent properties of Py-BODIPY and the localization of the dye inside the nanoparticles.

\section{Results and discussion}

\subsection{Pyrene-modified BODIPY (Py-BODIPY) synthesis and solvatochromic properties}

The typical emission of unsubstituted BODIPY chromophore falls in the region $470-530 \mathrm{~nm}$, but the position of the maximum of luminescence can be modulated by substitution at different positions of the BODIPY skeleton, and in particular of the meso-carbon atom. The strategy of functionalizing BODIPYs on the meso-carbon with polyaromatic rings such as naphthalene, ${ }^{34}$ anthracene, ${ }^{34}$ and phenanthrene, ${ }^{35}$ in order to improve

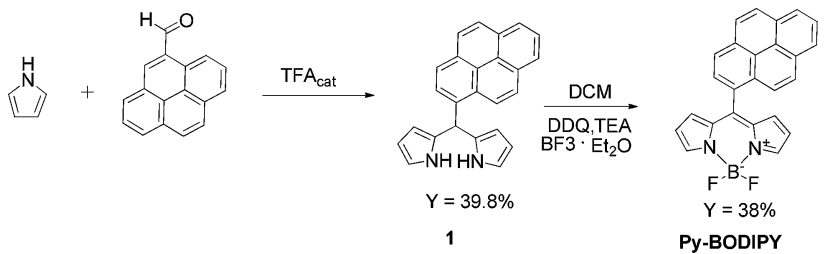

Scheme 1 Adopted synthetic procedure for the preparation of PyBODIPY.

the photophysical properties of the chromophore have been widely adopted.

In particular, the BODIPY derivative containing a pyrene group (Py-BODIPY) in the meso position was reported for the first time by Peña-Cabrera and co-workers ${ }^{36}$ using the LiebeskindSrögl cross-coupling reaction between 8-thiomethylBODIPY and pyrene boronic acid, catalyzed by copper(I) thiophene-2carboxylate (CuTC). Herein, we succeeded in synthesizing Py-BODIPY using the classic Lindsey method, ${ }^{37}$ i.e., acidcatalyzed condensation of 1-pyrenecarboxaldheide with pyrrole, followed by oxidation with DDQ and exposure to triethylamine and $\mathrm{BF}_{3} \cdot \mathrm{Et}_{2} \mathrm{O}$ (Scheme 1 and $\mathrm{ESI}^{\dagger}$ for synthetic details).

Although the method reported by Peña-Cabrera avoids the synthesis of dipyrromethanes precursors which might

Table 1 Quantum yield $(\Phi)$, excitation and emission wavelength ( $\lambda_{\text {exc }}$ and $\lambda_{\text {emis, }}$ respectively) of Py-BODIPY with different solvents

\begin{tabular}{llll}
\hline Solvent & $\lambda_{\text {exc }}(\mathrm{nm})$ & $\lambda_{\text {emis }}(\mathrm{nm})$ & $\Phi$ \\
\hline Hexane & 504 & 523 & 0.25 \\
Toluene & 507 & 582 & 0.14 \\
DCM & 505 & 670 & $2.0 \times 10^{-2}$ \\
THF & 504 & 630 & $4.0 \times 10^{-2}$ \\
EtOAc & 502 & 627 & $4.3 \times 10^{-3}$ \\
Acetone & 502 & 662 & $8.9 \times 10^{-3}$ \\
DMF & 505 & 670 & $6.1 \times 10^{-3}$ \\
MeCN & 499 & 708 & $4.9 \times 10^{-3}$ \\
DMSO & 507 & 709 & $9.5 \times 10^{-3}$ \\
EtOH & 502 & 651 & $6.8 \times 10^{-3}$ \\
MeOH & 501 & 662 & $5.6 \times 10^{-3}$
\end{tabular}

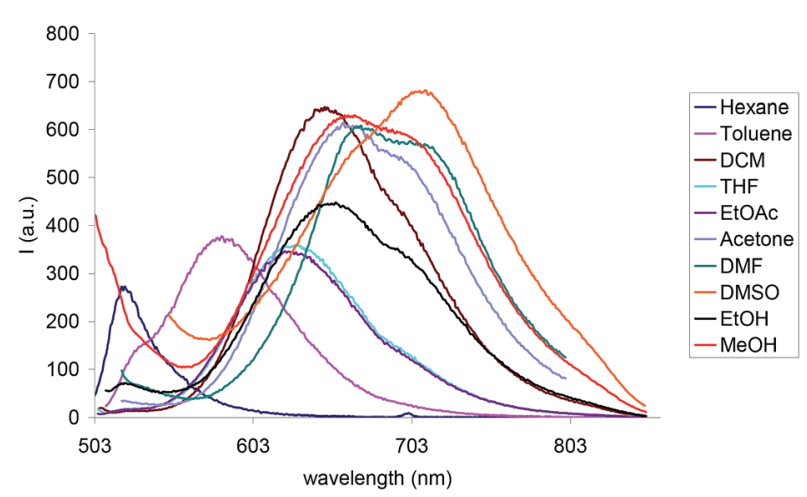

Fig. 1 Fluorescence spectra of Py-BODIPY in different solvents. [PyBODIPY] $=2.5 \times 10^{-5} \mathrm{M}$; $\lambda_{\text {exc }}$ between 499 and $507 \mathrm{~nm}$ (see Table 1). 
be difficult to purify, it requires the synthesis of both 8-thiomethylBODIPY and the catalyst. The Lindsey method is more straightforward to prepare Py-BODIPY as it requires two steps, being the 1-pyrenecarboxaldheide commercially available.

Here, we studied the photophysical properties of Py-BODIPY in different solvents covering a variety of polarities larger than that previously reported, ${ }^{38}$ and ranging from apolar hexane to polar DMSO (Table 1). While the absorption spectrum of Py-BODIPY showed negligible changes in the different solvents (ESI, Fig. S1 $\dagger$ ), its emission properties were strongly dependent on the polarity of the solvent, with the maximum of the fluorescence emission ranging from $523 \mathrm{~nm}$ in hexane to $709 \mathrm{~nm}$ in DMSO (Fig. 1).

\subsection{Nanoparticles physicochemical and photophysical characterization}

The hexosome nanoparticles investigated are engineered for theranostic applications in oncology. Therefore, to confer on them targeting properties towards tumor tissues, the stabilization of the nanoparticles was achieved using a 80/20 mixture of commercial PF108 and the folate-conjugate PF108 (PF108-FA). The synthesis of PF108-FA and its use for the formulation of targeted theranostic cubosomes were previously described in ref. 16. Since tumor cells often overexpress folate receptors, the folate molecules conjugated to the terminal PEO moieties of PF108 on the particle surface will actively favor the enrichment of nanoparticles at cancer cells compared to healthy cells. Hexosomes were also simultaneously loaded with Py-BODIPY, for optical imaging purposes, and camptothecin, an anticancer drug that, along with its analogues, was demonstrated to be effective in the treatment of a broad spectrum of tumors. The loading efficiency of the dye and the drug ( $E \%$, expressed as percentage of the amount of the drug and the dye present in the formulation before dialysis, see $\mathrm{ESI}_{\dagger}^{\dagger}$ ) were $97 \%$ and $54 \%$, respectively. The physicochemical characterization of the hexosome formulation containing both the diagnostic and the therapeutic agent (HexCBFA) was performed by DLS, SAXS, and cryo-TEM. For the sake of comparison the other targeting hexosome formulations, empty (HexFA) as well as those containing only the drug (HexCFA) or the dye (HexBFA), were also investigated by DLS (see ESI $\dagger$ for Experimental details).

In general, DLS results reported in Table 2 confirm that the 80/20 mixture of PF108/PF108-FA was able to stabilize the formulations as a nanometer-size particle dispersion with quite a low polydispersity and highly negative $\zeta$-potential. Moreover, encapsulation of the fluorophore and the drug, even when they are formulated simultaneously, did not cause appreciable alterations in the size, the PDI, and the $\zeta$-potential of the nanoparticles (Table 2).

The inner symmetry and the stability as a function of the temperature of the HexCBFA formulation were determined by synchrotron SAXS experiments. The diffraction patterns recorded at 25,37 , and $50{ }^{\circ} \mathrm{C}$ are reported in Fig. 2 . All of them are dominated by a strong reflection peak followed, at higher $q$ values, by two weaker reflections. The positions of these three Bragg peaks, in the ratio $1: \sqrt{ } 3: 2$, are always consistent with
Table 2 Average nanoparticle diameter $\left(D_{\text {av }}\right)$, polydispersity index (PDI), and $\zeta$-potential of various hexosome formulations. HexFA, indicate the hexosome formulation stabilized by the folate-conjugated PF108, while the capitol letters B and C in the acronyms indicate formulations loaded with Py-BODIPY and/or camptothecin, respectively

\begin{tabular}{llll}
\hline Formulation & $D_{\mathrm{av}}(\mathrm{nm})$ & PDI & $\zeta$-potential $(\mathrm{mV})$ \\
\hline HexFA & $168 \pm 1$ & 0.20 & $-33 \pm 1$ \\
HexCFA & $170 \pm 1$ & 0.20 & $-36 \pm 1$ \\
HexBFA & $177 \pm 1$ & 0.22 & $-32 \pm 1$ \\
HexCBFA & $172 \pm 1$ & 0.20 & $-35 \pm 1$ \\
\hline
\end{tabular}

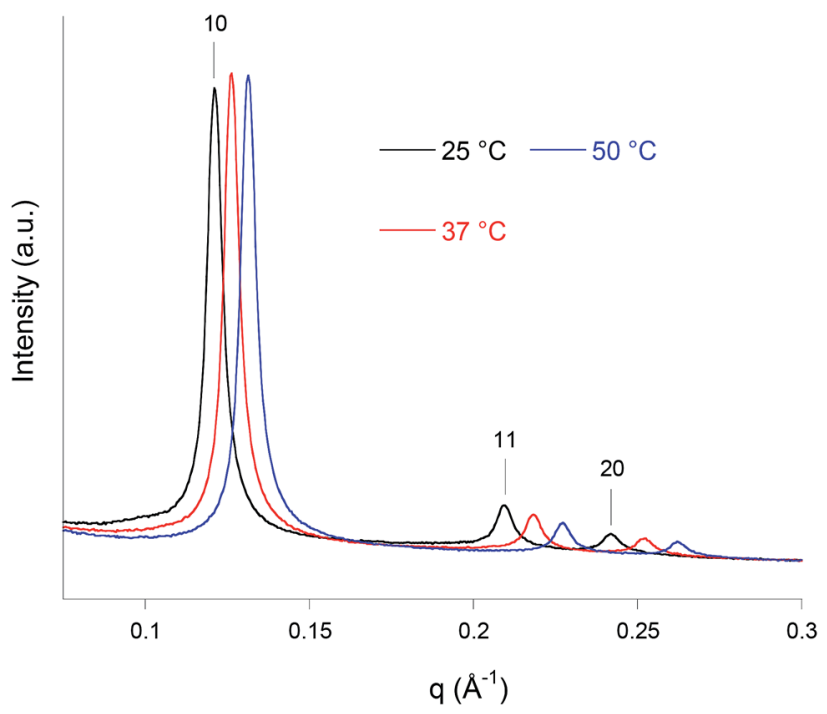

Fig. 2 Synchrotron SAXS diffraction patterns recorded at different temperatures of the hexosome formulation stabilized by the mixture PF108/folate-conjugated PF108 and loaded with Py-BODIPY and camptothecin (HexCBFA). The Miller indices ( $h k$ ) are reported on top of the corresponding Bragg reflections.

that expected for a reverse hexagonal phase. With temperature increasing, the lattice parameters, $a$, were found to be $59( \pm 1)$, $57( \pm 1)$, and $55( \pm 1) \AA$, respectively, while the corresponding radius of the water channels $r_{\mathrm{w}}$ were $31( \pm 1), 30( \pm 1)$, and $29( \pm 1)$ $\AA$, respectively. This is as generally observed for liquid crystalline systems. ${ }^{39}$ Nonetheless, the nanoparticles retain their internal reverse hexagonal symmetry even at the highest temperature $\left(50{ }^{\circ} \mathrm{C}\right)$.

Cryo-TEM observations of the same sample (Fig. 3) showed the presence of quasi-spherical nanoparticles characterized by curved striations and the absence of the small unilamellar vesicles usually found in cubosomes formulations, either attached to the nanoparticles or dispersed as single entities. Curved striations are a characterizing feature of the hexosomes, ${ }^{\mathbf{4 0 , 4 1}}$ and are related to the peculiar configuration adopted by the close (hexagonally) packed and deformable water tubes that constitutes the internal nanostructure. ${ }^{\mathbf{4 2}}$

From a photophysical point of view, when the HexCBFA formulation (dilution 1 : 30) was excited at $330 \mathrm{~nm}$ an emission band with the maximum centered at $532 \mathrm{~nm}$ (Fig. 4) was 


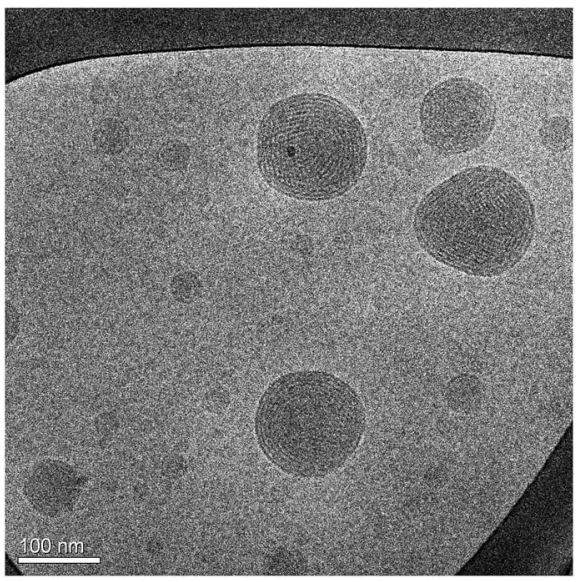

Fig. 3 Cryo-TEM image of the same sample investigated in Fig. 2.

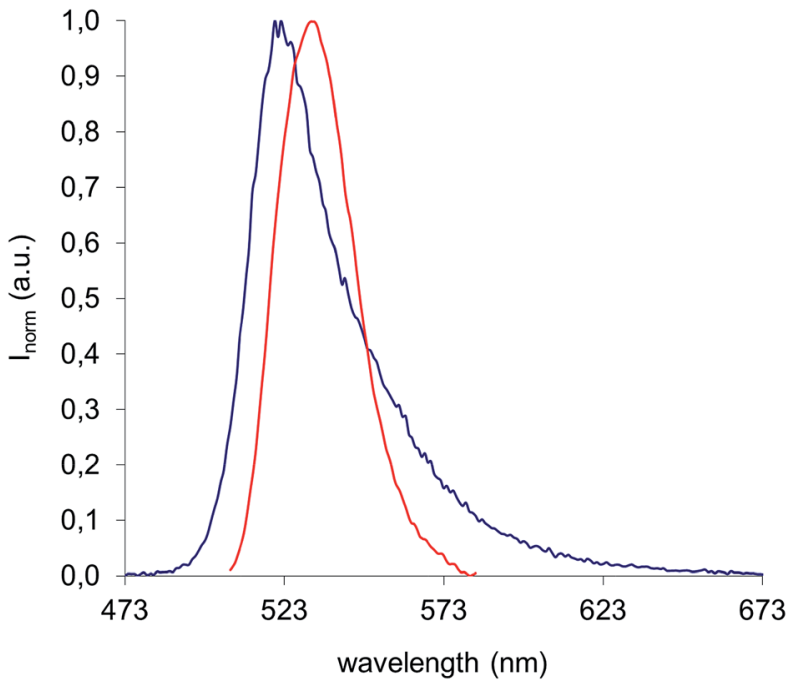

Fig. 4 Emission spectra of Py-BODIPY in hexane (blue curve) $\left(\lambda_{\text {exc }}=\right.$ $350 \mathrm{~nm}$ ) and of HexCBFA in water (dilution $1: 30$ ), $\lambda_{\text {exc }}=330$.

observed, a position quite similar to that observed for the fluorophore dissolved in hexane $\left(\lambda_{\text {emiss }}=523 \mathrm{~nm}\right)$.

The photostability of the HexCBFA formulation was tested by irradiating the sample under a UV-lamp $\left(\lambda_{\text {exc }}=365 \mathrm{~nm}\right)$ for $24 \mathrm{~h}$. No changes were observed in the emission properties of the formulation in these conditions.

\subsection{Internalization, viability, and Py-BODIPY localization against HeLa cells}

The uptake of the HexBFA nanoparticles was investigated by fluorescence microscopy (see ESI $\dagger$ for Experimental details). The image in Fig. 5 clearly shows that cytoplasmic fluorescent signals were observed in the cytoplasm of living cells after HexBFA nanoparticle incubation, whereas no fluorescence was detected in HexFA-treated cells. Remarkably, rather than a diffuse fluorescence the microscopic observations revealed intense fluorescent spots dispersed throughout the cytoplasm.

Given the shape and the size of these spots and the apolar nature of the dye, it could be inferred that the Py-BODIPY
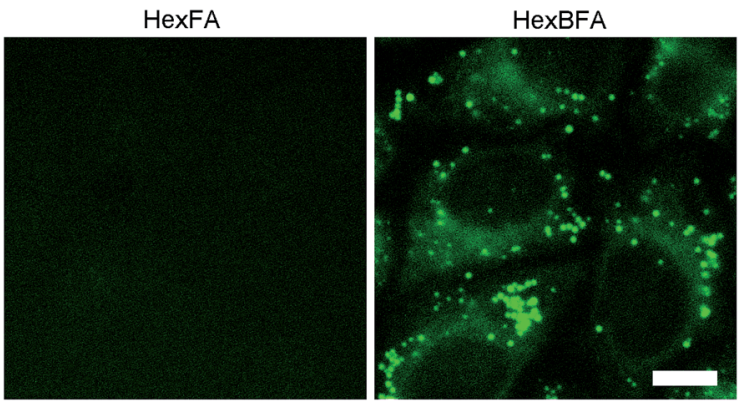

Fig. 5 Fluorescence microscopy of living HeLa cells treated with HexFA and HexBFA nanoparticles (incubated for 4 h) showing cytoplasmic fluorescence spots only inside the HexBFA-treated cells. Scale bar $=10 \mu \mathrm{m}$.

localizes in the lipid droplets. The latter are dynamic storage organelles that represent an intracellular reservoir of neutral lipids, such as triacylglycerols and cholesteryl esters, part of the lipid metabolism and used for membrane lipid synthesis. We recently demonstrated that monoolein-based nanoparticles (cubosomes) treatment induces intracellular accumulation of neutral lipids in the cytoplasmic lipid droplets. ${ }^{16}$ To investigate whether the Py-BODIPY fluorescence is localized in the lipid droplets, HexBFA-treated cells, grown on coverslips, were fixed and labeled with LipidTOX, a red neutral lipid stain with an extremely high affinity for neutral lipid droplets. This treatment resulted in the intracellular appearance of a colocalized Py-BODIPY (green) and LipidTOX (red) fluorescence, as shown
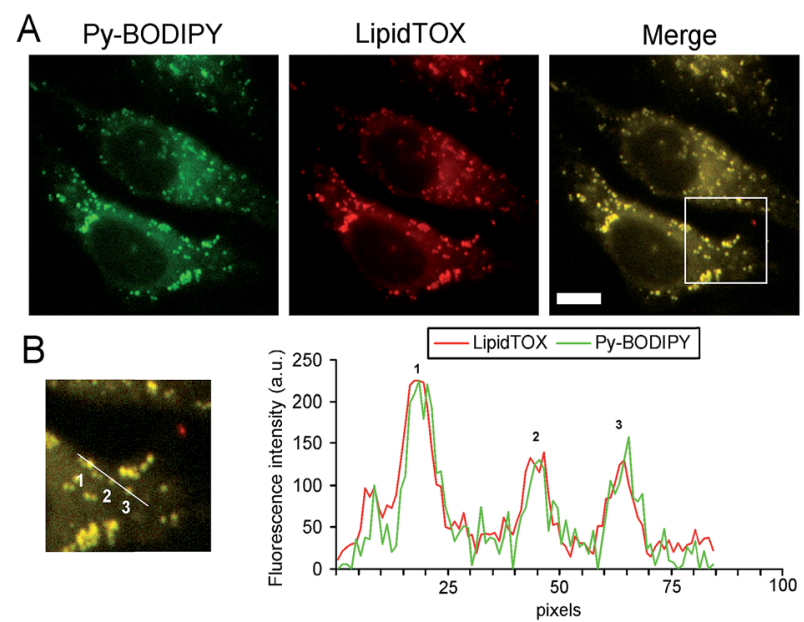

Fig. 6 Colocalization of Py-BODIPY with LipidTOX Red Neutral Lipid Stain. HeLa cells were treated with HexBFA nanoparticles (incubated for 4 h), fixed with 4\% PFA and stained with LipidTOX. (A) Cells are displayed in separate images showing the fluorescence of Py-BODIPY (green), LipidTOX (red) and in a composite image (colocalization in yellow). Scale bar $=10 \mu \mathrm{m}$. (B) Magnification of the square in the merged image as shown in (A) and fluorescent intensity profiles of PyBODIPY and LipidTOX. The linear plot (white line), sampled crossing over the cell cytoplasm and three lipid droplets, shows the fluorescence intensity of Py-BODIPY-green and LipidTOX-red fluorescence. The Py-BODIPY profile colocalizes perfectly with the LipidTOX profile thus indicating that Py-BODIPY stains neutral lipids in the lipid droplets. 


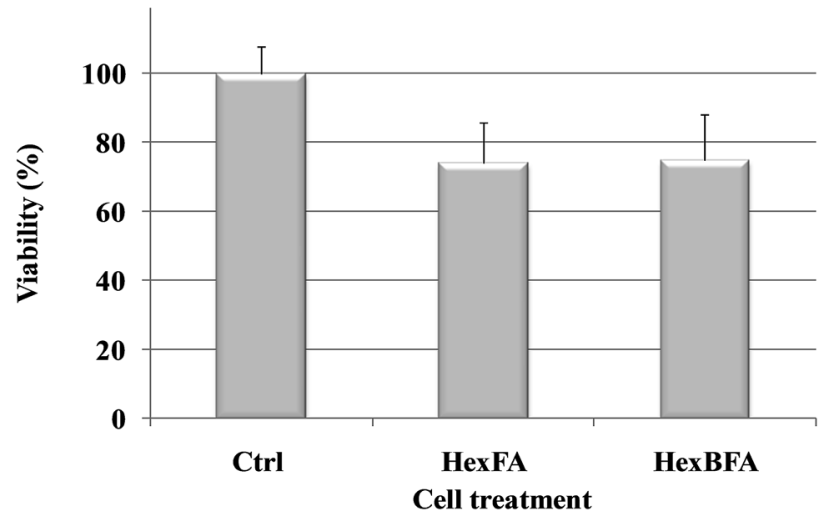

Fig. 7 Viability, expressed as \% of the control, induced by incubation for $4 \mathrm{~h}$ with HexFA and HexBFA nanoparticles $\left(1: 500,2 \mu \mathrm{L} \mathrm{mL}{ }^{-1}\right)$ in HeLa cells culture. Results were expressed as a mean \pm standard deviation (SD) of three independent experiments involving quadruplicate analyses for each sample.

by yellow fluorescence in the merged image and by intensity fluorescence profiles (Fig. 6).

The two nanoparticle formulations, HexFA and HexBFA, were also tested for cytotoxicity (MTT assay) in HeLa cells. Fig. 7 shows the viability, expressed as \% of the control, induced in HeLa cells after $4 \mathrm{~h}$ incubation. Evidently, treatments with both nanoparticle formulations did not induce a relevant reduction in cell viability as compared with control cells (see ESI $\uparrow$ for Experimental details).

\subsection{Theoretical calculations}

In order to correlate the photophysical properties of Py-BODIPY and the environment experienced by the dye in the formulation, theoretical calculations were carried out at Density Functional Theory (DFT) level. The effect of implicit solvation on the optimised metric parameters (Fig. 8; ESI, Table S1†) was evaluated at IEF-PCM level by considering the same solvents investigated in paragraph 2.1 (see also Table 1). In all cases, the optimised metric parameters were only marginally affected (see ESI $\dagger$ for Experimental details).

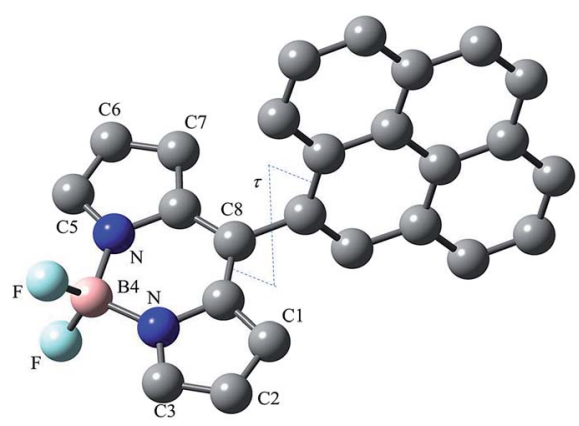

Fig. 8 Ball and stick drawing of the structure of Py-BODIPY optimised at DFT level in the gas phase. The numbering scheme for the BODIPY core is also reported. Hydrogen atoms have not been depicted for clarity.

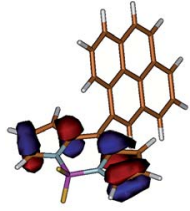

(a)

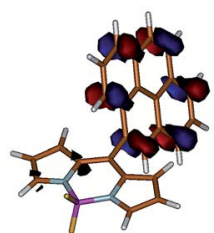

(b)

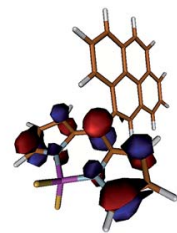

(c)
Fig. 9 Molecular orbital isosurfaces of HOMO-1 (a), HOMO (b), and LUMO (c) calculated for Py-BODIPY. Cutoff value 0.05 e.

In agreement with what was reported previously, ${ }^{38}$ the HOMO -1 and the LUMO are $\pi$-orbitals located on the BODIPY moiety, while the HOMO is centred on the pyrenyl substituent (Fig. 9), both in the gas phase and in solution.

Time-Dependent (TD) DFT calculations show that the singlet electron transition at the lowest energy S0 $\rightarrow \mathrm{S} 1$ (about $2.2 \mathrm{eV}$, Table S1 and Fig. S3, ESI $\dagger$ ) calculated for Py-BODIPY arises entirely from a HOMO $\rightarrow$ LUMO monoelectronic excitation, corresponding to a charge transfer (CT) process between the pyrene and the indacene systems. Due to the $\pi$-nature of the molecular orbitals (Fig. 9), in order for the overlap integral between the wavefunctions of HOMO and LUMO to be different from zero, the deviation of $\tau$ (the rotation of the pyrenyl ring, see $\mathrm{ESI} \uparrow$ for Experimental details) from orthogonality is required. Accordingly, this transition was calculated to be forbidden when the pyrene and the indacene planes were forced to be perpendicular. As a consequence of the CT nature of the transition, the S0 $\rightarrow$ S1 transition, which shows a very low oscillator strength at the optimised geometry, is calculated to be moderately solvatochromic in character (with a variation $\Delta \lambda$ in the calculated absorption wavelength of about $25 \mathrm{~nm}$ on passing from toluene to DMF).

The S0 $\rightarrow$ S2 transition (falling at about $3.0 \mathrm{eV}$, see Table S1 and Fig. S3, ESI $\dagger$ ) is instead assigned to a HOMO-1 $\rightarrow$ LUMO excitation. Due to its non-polar character, this transition is independent of the solvent $(\Delta \lambda=5 \mathrm{~nm})$. Although difference in the excitation energies between the two transitions (about $0.8 \mathrm{eV}$, see Table S1, ESI $\dagger$ ) might be overestimated by TDDFT calculations because of their difference in polarity, the structured experimental absorption band at about $500 \mathrm{~nm}$ can be attributed to the convolution of the transitions leading to the S1 and S2 excited states. TDDFT calculations allow accounting for the fluorescence of Py-BODIPY. Indeed, it is conceivable that the UV excitation from S0 to a Sk excited state is followed by an internal conversion to the S2 excited state. The possibility of the pyrene group to rotate (the libration vibration is calculated at about $20 \mathrm{~cm}^{-1}$ for the ground state), shown by the PES analysis (see ESI and Fig. S2 $\dagger$ ), could be responsible for the vibrational internal conversion S2 $\rightarrow$ S1. Hence, fluorescence emission would involve the S1 $\rightarrow$ S0 radiative relaxation. From a qualitative point of view, the polar nature of S1 nicely accounts for the remarkable solvatochromism of the emission discussed above. Indeed, in polar solvents (such as DMF, alcohols, acetonitrile) the $\mathrm{S} 1$ singlet excited state is stabilised $(E<2.2 \mathrm{eV}$, Table S1 and Fig. S3, ESI $\dagger$ ) by up to $0.1 \mathrm{eV}$ with respect to nonpolar solvents (hexane, toluene), so that emission is 
expected to occur at lower energies, as found experimentally (Fig. 1). Accordingly, the fluorescent emission observed in the hexosomes, similar to that detected in hexane, reflects the nonpolar nature of the environment experienced by the imaging agent (the hydrophobic MO chains), strongly supporting the localization of the Py-BODIPY within the bilayer.

\section{Conclusions}

In this paper we showed that nanoparticles with a $2 \mathrm{D}$ hexagonal nanostructure can be loaded with a pyrene-modified BODIPY fluorophore and the antineoplastic drug camptothecin and, at the same time, stabilized using a mixture of PF108 and folateconjugated PF108, so that they possess targeting properties toward tumor cells. The striking solvatochromism of the modified BODIPY, emitting at energies directly dependent on the polarity of the chemical environment, and the QM calculations performed, confirm the localization of the imaging agent within the bilayer. Moreover, we demonstrated that these nanoparticles are easily internalized by HeLa cells and that the fluorophore localizes within the lipid droplets. The cytotoxicity experiments, performed using the HexBFA formulation at a concentration of $75 \mu \mathrm{g} \mathrm{mL} \mathrm{mL}^{-1}$ of the dispersed phase $(\mathrm{MO}+$ block copolymers, or $68 \mu \mathrm{g} \mathrm{mL} \mathrm{m}^{-1}$ of $\mathrm{MO}$ ), the same as used for the fluorescence microscopy experiments, proved that the proposed formulation can be safely used for cellular imaging purposes. Remarkably, these results are in good agreement with our previous cytotoxicity tests performed on HeLa cells using cubosomes formulations, and those obtained by other groups working on similar nanoparticles and different cellular lines. ${ }^{43,44}$

Integration of therapeutics and imaging agents within a single theranostic nanoparticle allows monitoring drug delivery, release, and efficacy, contributing to realize tailormade therapies that, besides the study of genetic variations and biomarkers, exploit the development of imaging methods for predicting and evaluating therapeutic responses. ${ }^{45}$ Therefore, results here presented encourage the use of these nanoparticles in theranostic nanomedicine for personalized diagnosis and treatment of tumors.

\section{Acknowledgements}

We would like to thank Regione Autonoma della Sardegna (CRP-59699) for funding. The cryo-TEM work was performed at the Technion Laboratory for Electron Microscopy of Soft Matter, supported by the Technion Russell Berrie Nanotechnology Institute (RBNI).

\section{Notes and references}

1 S. T. Hyde, J. Phys. Chem., 1989, 93, 1458-1464.

2 K. Larsson, Nature, 1983, 304, 664.

3 K. Larsson, J. Phys. Chem., 1989, 93, 7304-7314.

4 S. Hyde, S. Andersson, K. Larsson, Z. Blum, T. Landh, S. Lidin and B. W. Ninham, The Language of Shape, Elsevier, Amsterdam, 1997.
5 Z. A. Almsherqi, S. D. Kohlwein and Y. Deng, J. Cell Biol., 2006, 173, 839-844.

6 Z. A. Almsherqi, T. Landh, S. D. Kohlwein and Y. Deng, Int. Rev. Cell Mol. Biol., 2009, 274, 275-342.

7 S. Murgia, S. Lampis, P. Zucca, E. Sanjust and M. Monduzzi, J. Am. Chem. Soc., 2010, 132, 16176-16184.

8 F. Caboi, G. S. Amico, P. Pitzalis, M. Monduzzi, T. Nylander and K. Larsson, Chem. Phys. Lipids, 2001, 109, 47-62, and references therein.

9 M. Monduzzi, S. Lampis, S. Murgia and A. Salis, Adv. Colloid Interface Sci., 2014, 205, 48-67.

10 S. Murgia, F. Caboi and M. Monduzzi, Chem. Phys. Lipids, 2001, 110, 11-17.

11 M. Carboni, A. M. Falchi, S. Lampis, C. Sinico, M. L. Manca, J. Schmidt, Y. Talmon, S. Murgia and M. Monduzzi, Adv. Healthcare Mater., 2013, 2, 692-701.

12 S. Murgia, A. M. Falchi, M. Mano, S. Lampis, R. Angius, A. M. Carnerup, J. Schmidt, G. Diaz, M. Giacca, Y. Talmon and M. Monduzzi, J. Phys. Chem. B, 2010, 114, 3518-3525.

13 R. Angelico, M. Carboni, S. Lampis, J. Schmidt, Y. Talmon, M. Monduzzi and S. Murgia, Soft Matter, 2013, 9, 921-928.

14 T. Landh, J. Phys. Chem., 1994, 98, 8453-8467.

15 X. Mulet, B. J. Boyd and C. J. Drummond, J. Colloid Interface Sci., 2013, 393, 1-20.

16 C. Caltagirone, A. M. Falchi, S. Lampis, V. Lippolis, V. Meli, M. Monduzzi, L. Prodi, J. Schmidt, M. Sgarzi, Y. Talmon, R. Bizzarri and S. Murgia, Langmuir, 2014, 30, 6228-6236.

17 S. Deshpande, E. Venugopal, S. Ramagiri, J. R. Bellare, G. Kumaraswamy and N. Singh, ACS Appl. Mater. Interfaces, 2014, 6, 17126-17133.

18 B. W. Muir, D. P. Acharya, D. F. Kennedy, X. Mulet, R. A. Evans, S. M. Pereira, L. K. Wark, B. J. Boyd, T.-H. Nguyen, T. M. Hinton, J. L. Waddington, N. Kirby, D. K. Wright, H. X. Wang, F. G. Egan and B. A. Moffat, Biomaterials, 2012, 33, 2723.

19 S. Murgia, S. Bonacchi, A. M. Falchi, S. Lampis, V. Lippolis, V. Meli, M. Monduzzi, L. Prodi, J. Schmidt, Y. Talmon and C. Caltagirone, Langmuir, 2013, 29, 6673-6679.

20 C. Nilsson, B. Barrios-Lopez, A. Kallinen, P. Laurinmäki, S. J. Butcher, M. Raki, J. Weisell, K. Bergström, S. W. Larsen, J. Østergaard, C. Larsen, A. Urtti, A. J. Airaksinen and A. Yaghmur, Biomaterials, 2013, 34, 8491-8503.

21 M. S. Muthu, D. T. Leong, L. Mei and S.-S. Feng, Theranostics, 2014, 4, 660-677.

22 K. Pombo García, K. Zarschler, L. Barbaro, J. A. Barreto, W. O'Malley, L. Spiccia, H. Stephan and B. Graham, Small, 2014, 10, 2516-2529.

23 J. A. Barreto, W. O'Malley, M. Kubeil, B. Graham, H. Stephan and L. Spiccia, Adv. Mater., 2011, 23, H18.

24 X. Zhang, S. Wang, M. Liu, L. Tao and Y. Wei, Nanoscale, 2013, 5, 147-150.

25 X. Zhang, X. Zhang, B. Yang, Y. Zhang and Y. Wei, ACS Appl. Mater. Interfaces, 2014, 6, 3600-3606.

26 A. Erten, W. Wrasidlo, M. Scadeng, S. Esener, R. M. Hoffman, M. Bouvet and M. Makale, Nanomedicine, 2010, 6, 797-807. 
27 Q. M. Kainz, A. Schätz, A. Zöpfl, W. J. Stark and O. Reiser, Chem. Mater., 2011, 23, 3606-3613.

28 G. Kang, H. Son, J. M. Lim, H. S. Kweon, I. S. Lee, D. Kang and J. H. Jung, Chem.-Eur. J., 2012, 18, 5843-5847.

29 T. I. Kim, J. Park and Y. Kim, Chem.-Eur. J., 2011, 17, 1197811982.

30 H. Y. Lee, D. R. Bae, J. C. Park, H. Song, W. S. Han and J. H. Jung, Angew. Chem., Int. Ed., 2009, 48, 1239-1243.

31 J. S. Lu, H. Fu, Y. Zhang, Z. J. Jakubek, Y. Tao and S. Wang, Angew. Chem., Int. Ed., 2011, 50, 11658-11662.

32 J. H. Olivier, J. Widmaier and R. Ziessel, Chem.-Eur. J., 2011, 17, 11709-11714.

33 K. M. L. Taylor-Pashow, J. Della Rocca, Z. Xie, S. Tran and W. Lin, J. Am. Chem. Soc., 2009, 131, 14261-14263.

34 H. Sunahara, Y. Urano, H. Kojima and T. Nagano, J. Am. Chem. Soc., 2007, 129, 5597-5604.

35 A. C. Benniston, A. Harriman, V. L. Whittle and M. Zelzer, Eur. J. Org. Chem., 2010, 2010, 523-530.

36 E. Peña-Cabrera, A. Aguilar-Aguilar, M. GonzálezDomínguez, E. Lager, R. Zamudio-Vázquez, J. GodoyVargas and F. Villanueva-García, Org. Lett., 2007, 9, 39853988.
37 R. W. Wagner and J. S. Lindsey, Pure Appl. Chem., 1996, 68, 1373-1380.

38 J. Bañuelos, I. J. Arroyo-Córdoba, I. Valois-Escamilla, A. Alvarez-Hernández, E. Peña-Cabrera, R. Hu, B. Zhong Tang, I. Ixone Esnal, M. Martíneza and I. López Arbeloa, RSC Adv., 2011, 1, 677-684.

39 H. Qiu and M. Caffrey, Biomaterials, 2000, 21, 223-234.

40 I. Amar-Yuli, E. Wachtel, E. B. Einav Ben Shoshan, D. Danino, A. Aserin and N. Garti, Langmuir, 2007, 23, 3637-3645.

41 M. Johnsson, Y. Lam, J. Barauskas and F. Tiberg, Langmuir, 2005, 21, 5159-5165.

42 L. Sagalowicz, M. Michel, M. Adrian, P. Frossard, M. Rouvet, H. J. Watzke, A. Yaghmur, L. De Campo, O. Glatter and M. E. Leser, J. Microsc., 2006, 221, 110-121.

43 N. Bye, O. E. Hutt, T. M. Hinton, D. P. Acharya, L. J. Waddington, B. A. Moffat, D. K. Wright, H. X. Wang, X. Mulet and B. W. Muir, Langmuir, 2014, 30, 8898-8906.

44 T. M. Hinton, F. Grusche, D. Acharya, R. Shukla, V. Bansal, L. J. Waddington, P. Monaghana and B. W. Muir, Toxicol. Res., 2014, 3, 11-22.

45 T. Lammers, S. Aime, W. E. Hennink, G. Storm and F. Kiessling, Acc. Chem. Res., 2011, 44, 1029-1038. 